\title{
Unveiling Gargantua: A new search strategy for the most massive central cluster black holes
}

\author{
M. Brockamp ${ }^{1}$, H. Baumgardt ${ }^{2}$, S. Britzen ${ }^{1}$, and A. Zensus ${ }^{1}$ \\ 1 Max Planck Institut für Radioastronomie, Auf dem Hügel 69, 53121 Bonn, Germany \\ 2 School of Mathematics and Physics, University of Queensland, St. Lucia, QLD 4072, Australia \\ e-mail: h. baumgardt@uq.edu.au
}

Received 1 July 2015 / Accepted 13 September 2015

\begin{abstract}
Aims. We aim to unveil the most massive central cluster black holes in the Universe.

Methods. We present a new search strategy, which is based on a black hole mass gain sensitive calorimeter and which links the innermost stellar density profile of a galaxy to the adiabatic growth of its central supermassive black hole (SMBH). As a first step we convert observationally inferred feedback powers into SMBH growth rates using reasonable energy conversion efficiency parameters, $\epsilon$. In the main part of this paper we use these black hole growth rates, sorted in logarithmically increasing steps encompassing our whole parameter space, to conduct $N$-body computations of brightest cluster galaxies (BCGs) with the newly developed MUESLI software. For the initial setup of galaxies, we use core-Sérsic models to account for SMBH scouring.

Results. We find that adiabatically driven core regrowth is significant at the highest accretion rates. As a result, the most massive black holes should be located in BCGs with less pronounced cores when compared to the predictions of empirical scaling relations, which are usually calibrated in less extreme environments. For efficiency parameters $\epsilon<0.1$, BCGs in the most massive, relaxed, and X-ray luminous galaxy clusters might even develop steeply rising density cusps. Finally, we discuss several promising candidates for follow-up investigations, among them the nuclear black hole in the Phoenix cluster. Based on our results, its central black hole might have a mass of the order of $10^{11} M_{\odot}$.
\end{abstract}

Key words. black hole physics - accretion, accretion disks - methods: numerical - galaxies: elliptical and lenticular, cD quasars: supermassive black holes - galaxies: clusters: general

\section{Introduction}

The detection of supermassive black holes (SMBHs) at the highmass end of the mass scale is of tremendous importance for constraining limitations of empirical scaling relations and thus for the understanding of the related evolution and growth of both SMBHs and cosmic structures like galaxies, galaxy groups, or even clusters of galaxies.

Black hole scaling relations link different properties of galaxies, such as total luminosity and bulge mass (Kormendy \& Richstone 1995; McLure \& Dunlop 2002; Marconi \& Hunt 2003; Häring \& Rix 2004; McConnell \& Ma 2013), the velocity dispersion, $\sigma$, (Ferrarese \& Merritt 2000; Gebhardt et al. 2000; Gültekin et al. 2009; McConnell \& Ma 2013), and the central surface brightness profile (Lauer et al. 2007a; Kormendy \& Bender 2009; Rusli et al. 2013; Dullo \& Graham 2014) to the mass of the nuclear SMBH. Currently, there is a debate about whether the empirical $M_{\bullet}-\sigma$ relation is a consequence of black hole feedback regulated galaxy growth (e.g., Silk \& Rees 1998; Fabian 2012) or results from stochastic averaging over numerous galaxy coalescences in a cosmological merger tree (Jahnke \& Macciò 2011)

The action of binary or multiple black holes after merger events of their host galaxies is suspected to be responsible for the formation of shallow central brightness profiles of luminous galaxies (Ebisuzaki et al. 1991; Milosavljević \& Merritt 2001; Merritt 2006) with $V$-band magnitudes $M_{V} \lesssim-21$ (Lauer et al. 2007b). This scenario is supported by Thomas et al. (2014), who found a tangentially biased velocity distribution within the break radius, $R_{\text {break }}$, which is located at the maximal curvature of the surface brightness profile. In the picture of SMBH merging, $R_{\text {break }}$ forms out, along with a tangentially biased velocity distribution, by the preferential scattering/removal of stars on eccentric orbits with low angular momentum. Therefore, the tight relationship, as indicated by scaling relations between $M$. and properties of the central galactic brightness profile, seems reasonable.

In a few particular and extreme cases, like MS0735 and Abell 85 (McNamara et al. 2009; López-Cruz et al. 2014), scaling relations that are usually calibrated in local and "ordinary" galaxies yield widely different black hole mass predictions by factors of 10-100. Using the $M_{\bullet}-R_{\text {break }}$ (Rusli et al. 2013), $M_{\bullet}-R_{\text {cusp }}$ (Lauer et al. 2007a) and $M_{\bullet}-L_{\text {def }}$ (Kormendy \& Bender 2009) relations, where $R_{\text {cusp }}$ is the cusp radius of a Nuker profile and $L_{\mathrm{def}}$ is the total luminosity difference between measured and extrapolated light profile, McNamara et al. (2009) and López-Cruz et al. (2014) found indications for extraordinary massive SMBHs. The central black hole masses in MS0735 and Abell 85 might be as high as $10^{11} M_{\odot}$ as long as their shallow density profiles are carved out by black hole merging processes ${ }^{1}$. The estimates of these three scaling relations are significantly above the predictions of the $M_{\bullet}-\sigma$ and $M_{\bullet}-L$ relations. There is tentative evidence that the $M_{\bullet}-\sigma$ (and $M_{\bullet}-L$ ) relation loses its predictive power (through a vertical tilt) above $\sigma=270 \mathrm{~km} \mathrm{~s}^{-1}$ (Kormendy \& Ho 2013).

\footnotetext{
1 At least for the BCG in Abell 85, Bonfini et al. (2015) show that the central brightness profile can be approximated by a Sérsic model with a small index $n$ and without a depleted core.
} 
Unrelated studies also give rise to skepticism concerning the validity of the $M_{\bullet}-\sigma$ and $M_{\bullet}-L$ relation when dealing with the most extreme central cluster galaxies: (i) via the fundamental plane of accreting black holes, Hlavacek-Larrondo et al. (2012a) found evidence for the presence of ultramassive black holes $\left(M_{\bullet} \geq 10^{10} M_{\odot}\right)$, which are $\approx 10$ times more massive than predictions based on the $M_{\bullet}-\sigma$ and $M_{\bullet}-L$ relations. Furthermore, (ii) the case for exceptionally massive black holes is strengthened by active galactic nuclei (AGN) with quasar like powers in form of mechanical feedback, but without any detectable $\mathrm{X}$-ray emission. The central active black holes in these cool core galaxy clusters must operate at highly sub-Eddington accretion rates to avoid detection and are most likely ultramassive (Hlavacek-Larrondo \& Fabian 2011).

Central cluster galaxies, which are located in the most massive (cool core) galaxy clusters, are therefore promising candidates for hosting the largest black holes in the Universe. Indeed, stellar dynamical mass measurements of even less extreme BCGs in the nearby Coma ${ }^{2}$ and Leo galaxy cluster, as well as lenticular galaxies, unveiled black holes around $M_{\mathbf{0}}=$ $10^{10} M_{\odot}$ (McConnell et al. 2011; van den Bosch et al. 2012).

This paper investigates the impact of hot cluster gas accretion on the dynamics and density profiles of BCGs. We relate the old idea of adiabatically growing black holes (Young 1980) in combination with core depletion by merging SMBHs to create a tool we call a black hole mass gain sensitive calorimeter. This tool offers a way to test SMBH accretion models, improve the understanding of scaling relations that depend on the surface brightness profile and help to unveil the most massive cluster black holes. If combined with the total feedback energy of a galaxy cluster, the inner slope and break radius, $R_{\text {break }}$, of its central cluster galaxy and SMBH mass, the calorimeter might be used to uniquely determine the SMBH mass gain during the early quasar era, through subsequent merging activity and accretion of hot cluster gas. Furthermore, it can be used to infer the time averaged binding energy conversion efficiency parameter, $\epsilon$.

In Sect. 2 we first compile observationally motivated black hole growth rates. These rates also cover the extremes, from a mere spin-powered feedback scenario $\left(\mathrm{d} M_{\bullet} / \mathrm{d} t \approx 0\right)$ up to SMBH growth with small $\epsilon$ in X-ray luminous galaxy clusters $\left(L_{\mathrm{X}}=10^{45}-10^{46} \mathrm{erg} \mathrm{s}^{-1}\right)$ like the Phoenix- (McDonald et al. 2012) or RX J1347.5-1145 cluster (Gitti \& Schindler 2004). In the main part of this paper (Sect. 3) we perform computations with the newly developed MUESLI software (Brockamp et al. 2014) to evaluate the impact of the (compiled) SMBH growth rates on the innermost surface brightness profiles. In Sect. 4 we discuss the obtained results, present a few cluster candidates that are recommended for follow-up observations, and describe future applications and improvements of the calorimeter. Our main findings are summarized in Sect. 5.

\footnotetext{
2 The Coma cluster neither hosts a cool core (Simionescu et al. 2013) nor is dominated by one central cluster galaxy. Instead, there are three dominating galaxies, which are NGC 4889, NGC 4874 and NGC 4839. They will likely coalesce sometime in the future (Gerhard et al. 2007). Their merger will give rise to a black hole of the order of $M_{\mathbf{0}} \approx$ $5 \times 10^{10} M_{\odot}$. For this calculation, we used direct SMBH mass estimates from Magorrian et al. (1998) for NGC 4874, McConnell et al. (2011) for NGC 4889 and obtained a mass of $M_{\bullet} \approx 6.5 \times 10^{9} M_{\odot}$ for NGC 4839 with the help of the $M_{\bullet}-R_{\text {break }}$ relation from Rusli et al. (2013) and a graphically evaluated break radius from Fig. 3 in Jordán et al. (2004). Mass loss through gravitational wave emission (Tichy \& Marronetti 2008) and stellar accretion in nonspherical potentials (Liu \& Chen 2013; Vasiliev 2014) is neglected.
}

\section{Accretion models}

In this section we present central cluster black hole growth models, which serve as the initial setup for our $N$-body computations in Sect. 3. They are obtained from the observed AGN feedback power in combination with reasonable accretion efficiency parameters (Sect. 2.1). Four mutually independent methods are reviewed in ascending order with regard to their mass accretion rate. As a result of the vast amount of literature, our compiled list is far from complete, but it covers the extreme scenarios. A consistency check is made in Sect. 2.3 by comparing the observationally motivated black hole growth rates with classical Bondi models to obtain reasonable initial black hole masses for our $N$-body computations.

\subsection{Conversion of feedback power into the SMBH growth rate}

The deposited AGN feedback (jet) power, $P_{\mathrm{AGN}}$, is converted into cluster black hole growth rates, $\dot{M}_{\bullet}$, using the relation

$\dot{M}_{\bullet}=\frac{\mathrm{d} M_{\bullet}}{\mathrm{d} t}=(1-\epsilon) \dot{M}_{\mathrm{acc}}=\frac{1-\epsilon}{\epsilon c^{2}} P_{\mathrm{AGN}}$.

Here, $\dot{M}_{\mathrm{acc}}=P_{\mathrm{AGN}} \epsilon^{-1} c^{-2}$ is the accretion rate and $\epsilon$ is the binding energy conversion efficiency parameter. To account for uncertainties related to $\epsilon$, we use the six efficiency parameters, $\epsilon=1.0 / 0.42 / 0.2 / 0.1 / 0.057 / 0.01$, which span over two orders of magnitude. They are motivated as follows:

- $\epsilon=1.0 / 0.1:$ these values are obtained from the electromagnetic jet efficiency formula, $\epsilon \approx 0.002 \times(1-|a|)^{-1}$ (Hawley $\&$ Krolik 2006) in combination with black hole spin values $a=0.998$ and $a=0.98$. While $a=0.998$ is the theoretical limit, which can be obtained in accretion processes (Thorne 1974), $a=0.98$ corresponds to the averaged spin of 48 luminous AGN at high redshift $(z \geq 1.5)$ with masses $M_{\bullet} \geq 3 \times 10^{9} M_{\odot}$ (Trakhtenbrot 2014). Their dimensionless spin parameter, $a=0.98$, is derived from deduced radiative efficiency parameters of individual accretion disks. Additionally, $\epsilon=0.1$ is often used as the canonical value for the energy conversion efficiency (e.g., Frank et al. 1992).

- $\epsilon=0.42 / 0.2 / 0.057$ : the value $\epsilon=0.057(\epsilon=0.42)$ is the maximal conversion efficiency of a (corotating) circular accretion flow (within the equatorial plane) around a nonspinning (maximally spinning) black hole (e.g., Novikov \& Frolov 1989). The parameter $\epsilon=0.2$ lies in between.

- $\epsilon=0.01$ : the kinetic jet/feedback efficiency parameter derived from AGN observations (Körding et al. 2008; Merloni \& Heinz 2008).

\subsection{Growth rate based on observational models}

Following the empirical relationship between mechanical work and jet luminosity at $1.4 \mathrm{GHz}$ from Cavagnolo et al. (2010), Ma et al. (2013) obtained a time and galaxy cluster averaged AGN power of $P_{\mathrm{AGN}}=3 \times 10^{44} \mathrm{erg} \mathrm{s}^{-1}$. The corresponding $\mathrm{SMBH}$ growth rate is

$\dot{M}_{\bullet \text { low }}=0.005\left(\frac{1-\epsilon}{\epsilon}\right) M_{\odot} \mathrm{yr}^{-1}$.

Equation (2) defines our low mass accretion model as it is strongly exceeded by AGN in some of the most massive cool core galaxy clusters, which are the most promising candidates for hosting the heaviest black holes. 
X-ray observations of these clusters demonstrate that the deposited AGN feedback power required to inflate X-ray cavities within the intra cluster gas (ICM) exceeds $P_{\mathrm{AGN}}=10^{45} \mathrm{erg} \mathrm{s}^{-1}$. Hlavacek-Larrondo et al. (2012b) report cavity powers as high as $P_{\mathrm{AGN}}=(4-8) \times 10^{45} \mathrm{erg} \mathrm{s}^{-1}$ for individual cases of massive galaxy clusters. Because of a duty cycle (i.e., fraction of time where the central cluster black hole is active) of at least $60-70 \%$ (Dunn \& Fabian 2006; Bîrzan et al. 2012), we assume a conservative value of $P_{\mathrm{AGN}}=3 \times 10^{45} \mathrm{erg} \mathrm{s}^{-1}$, which is also found in Hlavacek-Larrondo et al. (2015). This corresponds to

$\dot{M}_{\bullet \text { med }}=0.05\left(\frac{1-\epsilon}{\epsilon}\right) M_{\odot} \mathrm{yr}^{-1}$,

which defines our medium mass accretion model. The assumption of linear mass increase is justified by cavity energetics, which seemingly do not evolve with redshift (Hlavacek-Larrondo et al. 2012b).

But even the value defined in Eq. (3) has to be regarded as a lower limit. The feedback energy is neither fully deposited inside the cool core nor are cavities the only source of ICM heating. Energy can be transferred out to much larger radii via large scale shocks or sound waves. One spectacular example is the aforementioned system, MS0735, with its bipolar 200-240 kpc X-ray cavities surrounded by Mach 1.26 shocks waves (Vantyghem et al. 2014). They extend to at least twice the size of the cooling radius. Indeed, studies that calculate the total injected AGN feedback energies out to large radii obtain time-averaged jet powers as high as $P_{\mathrm{AGN}}=10^{46} \mathrm{erg} \mathrm{s}^{-1}$ (Mathews \& Guo 2011; Chaudhuri et al. 2012). These values are deduced from comparing observed potential energies of virialized massive clusters $\left(M_{\mathrm{vir}} \approx 10^{15} M_{\odot}\right)$ to those obtained from simulations without feedback. Following Mathews \& Guo (2011), the energetic demands and high accretion rates of these central cluster black holes cannot be lowered by splitting them into multiple sources. In most cases, X-ray cavities and shocks are associated with the central BCG and not its satellites. Significant preheating of baryons prior to galaxy cluster assembly is also disregarded by Mathews \& Guo (2011).

Following Churazov et al. (2002), similar (or even higher) AGN feedback powers are also required for balancing the selfcooling of the gas in the most X-ray luminous, cool-core clusters approaching $L_{\mathrm{X}} \approx 10^{46} \mathrm{erg} \mathrm{s}^{-1}$, such as MACSJ1447.4+0827 (Hlavacek-Larrondo et al. 2012b), RX J1347.5-1145 (Gitti \& Schindler 2004), or the Phoenix cluster (McDonald et al. 2012). Integrated over galaxy cluster lifetimes of seven to eight billion years and by assuming various efficiency parameters (from as high as unity for black hole spin-powered models down to a few percent for Schwarzschild black holes; see Sect. 2.1) this results in up to $\Delta M_{\bullet} \approx 10^{10} M_{\odot}$ of accreted mass. Feedback energies as high as $P_{\mathrm{AGN}}=10^{46} \mathrm{erg} \mathrm{s}^{-1}$ define our high mass accretion scenario, i.e.,

$\dot{M}_{\bullet \text { high }}=0.18\left(\frac{1-\epsilon}{\epsilon}\right) M_{\odot} \mathrm{yr}^{-1}$.

Central cluster black holes growing at these prodigious rates might pose a challenge for theories, which assume that significant SMBH growth ceased early on in accordance with cosmic downsizing of the AGN activity (Hasinger et al. 2005; Kelly \& Shen 2013). Alternatively, the feedback might be powered mainly by trapped spin energy $(\epsilon \approx 1)$ or central cluster black holes might have reached dimensions where even $\Delta M_{\bullet} \gtrsim$ $10^{9} M_{\odot}$ of accreted gas represents only a tiny fraction of their total mass (Hlavacek-Larrondo et al. 2012b). The latter idea

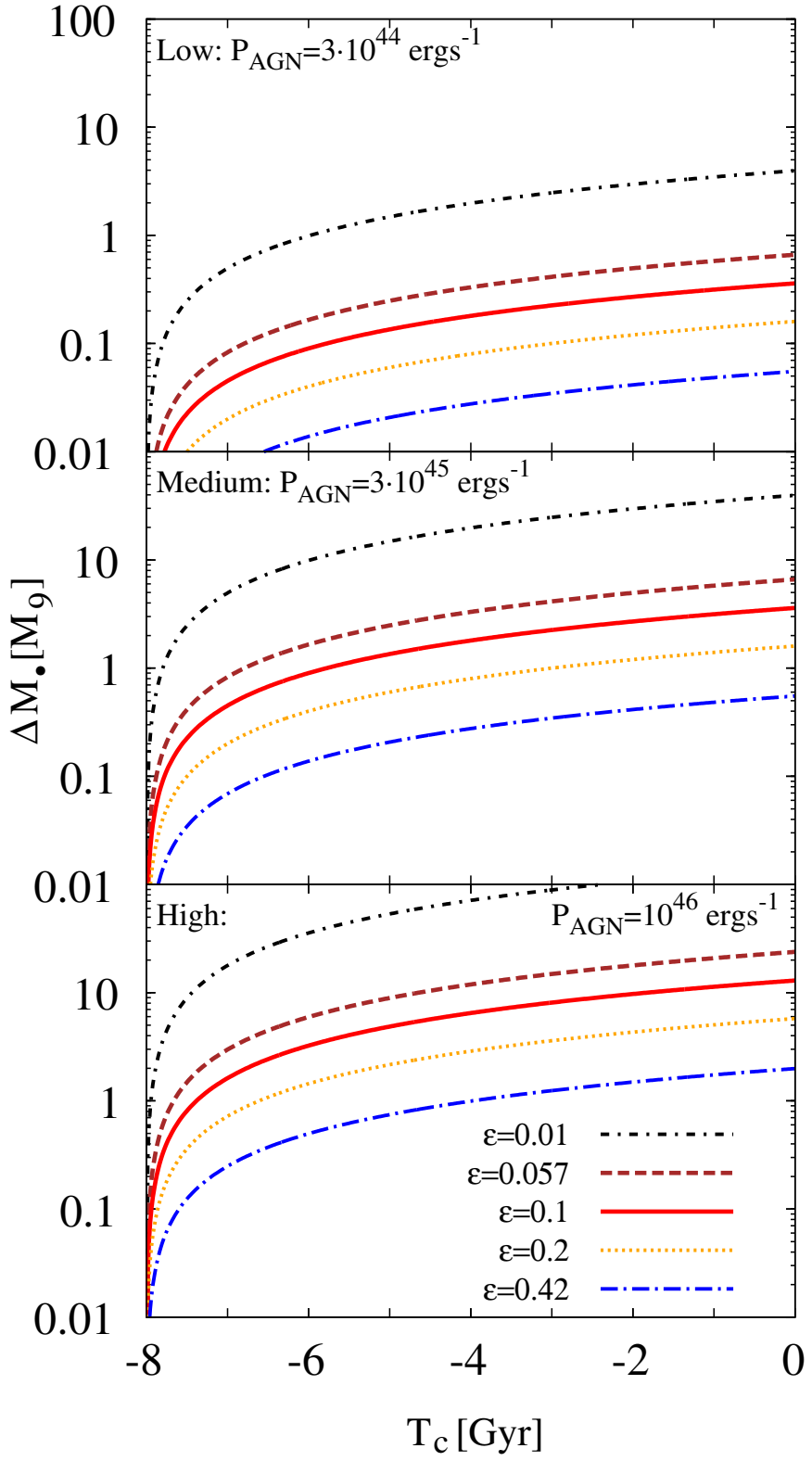

Fig. 1. The SMBH growth rate is obtained from the central cluster AGN feedback power, which is constrained by means of different strategies and cluster samples. The colors and line types indicate the binding energy conversion efficiency parameters, which must be adopted for the conversion (Eq. (1)). The cosmic time is defined as $T_{\mathrm{c}}(z=0)=0$, and we assume that feedback/accretion started operating at redshift $z=1$ ( 8 billion years ago). SMBH masses can be obtained from the relation $M_{\bullet}(t)=M_{\bullet \text { init }}+\Delta M_{\bullet}(t)$.

is in accordance with the existence of individual cases of extremely massive AGN in the early Universe (Ghisellini et al. 2009; Trakhtenbrot 2014; Banerji et al. 2015; Wu et al. 2015; Jun et al. 2015) and conforms with the upper limit of the black hole (quasar) mass function (BHMF; Kelly \& Shen 2013, their Chap. 3.3). The obtained BHMF is calibrated by the virial mass estimates of 58.000 Type 1 quasars.

The observation-based models are graphically illustrated in Fig. 1. Models with $\epsilon=1\left(\Delta M_{\bullet}=0\right)$ are not plotted subject to the logarithmic spacing of the $y$-axis. With regard to the enormous spread in $\epsilon$ (Sect. 2.1), the accumulated SMBH mass over 8 Gyr (i.e., the typical lifetime of galaxy clusters), varies from $\Delta M_{\bullet}=0$ up to several $10^{10} M_{\odot}$. Consequently, the growth 
of the SMBH cannot be uniquely constrained from the deposited feedback energy. Therefore, it is important to understand the influence of the growing black hole on its immediate surroundings. This leads us to the idea of the black hole mass gain sensitive calorimeter, which is part of Sect. 3.

\subsection{Consistency check: Classical Bondi models}

Through a comparison with theoretically justified models, we can explore whether the initial black hole masses, which are chosen for our $\mathrm{N}$-body computations and which cannot be directly deduced from the observed feedback powers, are realistic. We focus on galaxy clusters with relaxed gas atmospheres and ignore cold gas accretion scenarios subject to the absence of a positive correlation between jet power and molecular gas mass (McNamara et al. 2011). We assume that accretion flows are not disturbed through shock heating by collisions and only consider well-established models, i.e., classical Bondi flows (Bondi 1952) with some improvements from more recent investigations. The usage of sophisticated (analytical) models like radiating Bondi flows (Mathews \& Guo 2012) is left for future studies.

We show SMBH growth rates related to classical Bondi accretion models in Fig. 2. We do not consider the influence of the growing black hole on the accretion rate, and discuss some consequences this might have in Sect. 4.3. Our setup includes three initial black hole masses with $M_{\bullet}=1,2,4 \times 10^{10} M_{\odot}$. The highest value corresponds to the upper quasar mass obtained from the black hole mass function (Kelly \& Shen 2013). Individual cases of black holes with these masses at high redshift are also found in Trakhtenbrot (2014) and Jun et al. (2015) and are required to successfully model (but not strictly unambiguously) the spectral energy distribution and total luminosity of the blazar S5 $0014+813$ at $z=3.37$ (Ghisellini et al. 2009) ${ }^{3}$. The three initial SMBH masses are discriminated using different line types in Fig. 2.

Apart from some modifications, which are specified in the text, we make use of the same strategy as Allen et al. (2006) and use their equations and parameter setting for calculating the black hole growth rate through Bondi accretion,

$\dot{M}_{\bullet \mathrm{B}}=\eta(1-\epsilon) 4 \pi \lambda\left(G M_{\bullet}\right)^{2} c_{\mathrm{s}}^{-3} \rho$.

Here, $\eta=0.2$ is a conservative correction parameter for rotating and viscous gas atmospheres (Narayan \& Fabian 2011). The term $(1-\epsilon)=0.9$ guarantees energy conservation (assuming $\epsilon=0.1)$ and the coefficient $\lambda=0.25$ is related to the adiabatic index $\gamma=5 / 3$. The sound velocity at the characteristic Bondi or accretion radius, $R_{\mathrm{a}}=2 G M_{\bullet} / c_{\mathrm{s}}^{2}$, is $c_{\mathrm{s}}=\sqrt{\gamma k_{\mathrm{B}} T /\left(\mu m_{\mathrm{p}}\right)}$. The parameter $k_{\mathrm{B}}$ is the Boltzmann constant, $T$ is the gas temperature, $\mu=0.62$ is the average atomic weight, and $m_{\mathrm{p}}$ is the mass of the proton. The gas density, $\rho=1.13 n_{\mathrm{e}} m_{\mathrm{p}}$, which is also taken from Allen et al. (2006), can be calculated from the measurable electron density, $n_{\mathrm{e}}$.

The remaining free parameters, $n_{\mathrm{e}}$ and $T$, which are required for our growth models, are taken from a cluster sample average. To be more precise, we calculate the arithmetic mean values of $n_{\mathrm{e}}$ and $T$ at $R_{\mathrm{a}}$ from Table 4 in Russell et al. (2013). Their sample includes the same galaxy clusters as Allen et al. (2006) plus four additional objects. Two sets of parameters (distinguished with different colors in Fig. 2) are chosen. The first,

\footnotetext{
3 Blazars are highly beamed sources, so for every blazar there should be several nonbeamed AGN with similar properties (Ghisellini et al. 2009).
}

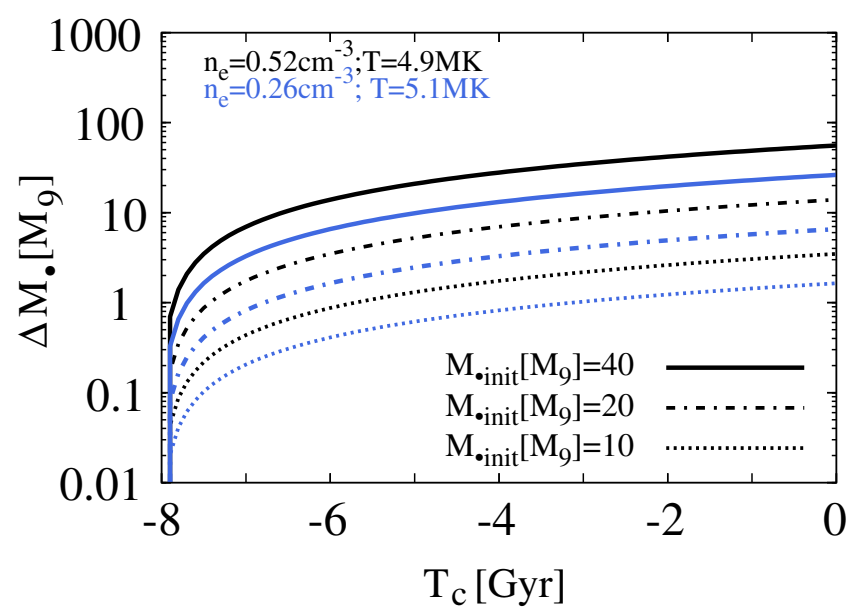

Fig. 2. Black hole growth models related to the classical Bondi accretion scenario. Although the rates differ widely and predict extreme upper values, they lie within the parameter range of the observation-based models, which are plotted in Fig. 1. In case of very massive seed black holes around $M_{\bullet}=10^{10} M_{\odot}$ at $z=1$, Bondi accretion alone is sufficient to explain the deposited feedback energies even in the most extreme cool core clusters.

$n_{\mathrm{e}}=0.52 \mathrm{~cm}^{-3}, T=4.9 \times 10^{6} \mathrm{~K}$, represents the mean, whereas the second, $n_{\mathrm{e}}=0.26 \mathrm{~cm}^{-3}, T=5.1 \times 10^{6} \mathrm{~K}$, is obtained from the upper $(T+\Delta T)$ and lower $\left(n_{\mathrm{e}}-\Delta n_{\mathrm{e}}\right) 1 \sigma$ mean values. With this approach, we tend to underestimate the true SMBH growth rate.

Uncertainties related to $R_{\mathrm{a}}$ (which depends on the assumed $\left.M_{\bullet}\right)$ and the parameters $n_{\mathrm{e}}\left(R_{\mathrm{a}}\right)$ and $T\left(R_{\mathrm{a}}\right)$ are compensated by a constant ratio $c_{\mathrm{s}}^{-3} \rho \propto P T^{-5 / 2}=$ const. (in Eq. (5)), which is independent of radius, $r$, for $\gamma=5 / 3, P=n k_{\mathrm{B}} T$ and $T^{\gamma} P^{1-\gamma}=$ const. It is only important that $n_{\mathrm{e}}$ and $T$ are measured deep within the potential of the central host galaxy because of its influence on the Bondi accretion flow.

Evidently, all analytical-based models, which are presented in Fig. 2 lie within the parameter range of the observation-based models (Sect. 2.2). This indicates that Bondi accretion alone is sufficient for powering the most extreme central cluster AGN hosting the largest SMBHs. Furthermore, it shows that initial black hole masses as high as $M_{\bullet}=(1-4) \times 10^{10} M_{\odot}$ at the onset of hot cluster gas accretion are reasonable.

\section{Cusp formation due to SMBH growth}

Initial conditions and results of $N$-body computations are presented in this section. The idea is to numerically investigate, for a sample of representative black hole growth models (Sect. 2), the adiabatic response of the red/old stellar density profile of the host galaxy to the mass, which becomes trapped by the black hole. Under the restriction that the duration of the SMBH growth phase is larger than the orbital timescale, the term adiabatic refers to the increase of the central density, while the angular momentum distribution simultaneously remains conserved. This idea goes back to Young (1980) and was later used by van der Marel (1999) to explain and predict the observed powerlaw cusps of early-type galaxies and central SMBH masses. van der Marel (1999) assumed that initial galaxies already started with constant density cores (similar to globular clusters without core-collapse) and concluded that less-luminous galaxies with steeply rising slopes are those galaxies which host the most massive black holes when normalized to the mass of the 
Table 1. Initial parameters of the model galaxies.

\begin{tabular}{cccccccc}
\hline \hline Model & $T[\mathrm{Gyr}]$ & Init. $M_{\bullet}\left[M_{9}\right]$ & $M_{\text {dyn }}\left[M_{9}\right]$ & $R_{\mathrm{e}}[\mathrm{kpc}]$ & $R_{\mathrm{b}}[\mathrm{kpc}]$ & $\Delta M_{\bullet}\left[M_{9}\right]$ & Comment \\
\hline 1 & 8 & 1.5 & 400 & 4 & 0.15 & 0.06 & OB-low, $\epsilon=0.42$ \\
2 & 8 & 5 & 770 & 7 & 0.4 & 0.17 & OB-low, $\epsilon=0.2$ \\
3 & 8 & 10 & 1550 & 18 & 0.8 & 0.38 & OB-low, $\epsilon=0.1$ \\
4 & 8 & 10 & 1550 & 18 & 0.8 & 0.7 & OB-low, $\epsilon=0.057$ \\
5 & 8 & 15 & 1750 & 25 & 1.3 & 4 & OB-medium, $\epsilon=0.1$ \\
6 & 8 & 20 & 1750 & 25 & 1.8 & 6 & OB-high, $\epsilon=0.2$ \\
7 & 8 & 20 & 1750 & 25 & 1.8 & 13 & OB-high, $\epsilon=0.1$ \\
8 & 8 & 40 & 2000 & 68 & 3.8 & 40 & OB-medium, $\epsilon=0.01$ \\
9 & 6.7 & 40 & 2000 & 68 & 3.8 & 120 & OB-high, $\epsilon=0.01$ \\
\hline
\end{tabular}

Notes. List of parameters selected for our models $/ N$-body computations. OB stands for observation-based models (Sect. 2.2). Slope values $\gamma=0.2$, $\alpha=4.25$, and $n=4$ (Eq. (6)) are used for all models. The huge initial SMBH masses in excess of $10^{10} M_{\odot}$ are motivated at the end of Sect. 2.2 and in Sect. 2.3. While dynamical masses, $M_{\mathrm{dyn}}$, and effective radii, $R_{\mathrm{e}}$, of our model galaxies are constrained by representative galaxies, the break-radius, $R_{\mathrm{b}}$, is taken from the scaling relation in Rusli et al. (2013). SMBH masses during the $N$-body computations are linearly increased up to $\Delta M_{\bullet}$ over the typical galaxy cluster lifetime of $T \approx 8$ Gyr. The $\Delta M_{\bullet}$ values are selected to equally cover (in log-space) the whole parameter range of SMBH growth rates, which are specified in Sect. 2. Model 9 is computed from $z=1$ until $z=0.1$.

prior constant density core. This leads to incorrect predictions (e.g., by a factor 30 for NGC 4889) because it underestimates the merger history of these galaxies. Instead, SMBH scouring leads in a natural way to the depletion of galactic centers (Ebisuzaki et al. 1991; Milosavljević \& Merritt 2001; Merritt 2006) and it predicts that the most massive black holes reside in galaxies with the most pronounced cores. Ravindranath et al. (2002) treated both scenarios (adiabatic and SMBH scouring) as competing models and argued that black hole merging is superior in explaining the observed parameter relations. Today, adiabatic black hole growth models have mostly disappeared from the literature ${ }^{4}$.

Motivated by the wide distribution of the central cluster black hole growth rates (Sect. 2), we hope to rehabilitate adiabatic SMBH growth models, but this time not as a competitor model to SMBH scouring. The relocation of mass, i.e., adiabatic black hole growth (Young 1980; Goodman \& Binney 1984; van der Marel 1999) leads to a cusp regeneration process of the depleted density centers after SMBH scouring. Of course, the obtained results depend on initial conditions and the accretion rate of hot cluster gas. In this sense, our findings are not free of systematics either, as we also have to make assumptions about initial conditions, which might turn out to be inappropriate. However, initial conditions can be modified in future studies. The method itself allows us to construct a black hole mass gain sensitive calorimeter, which is based on the innermost surface brightness profile and can be used to estimate (independently of $\epsilon$ ) the amount of swallowed mass in galaxy clusters.

As already motivated in Sect. 2.3, we must assume that black hole growth (through hot gas) is powered by accretion flows which extend far outward, e.g., classical Bondi or giant advection dominated flows. Accretion from a nearby molecular gas reservoir, which is not replenished cannot lead to adiabatic cusp regrowth, owing to an unchanged potential. Star formation, which can also increase the central surface brightness, is neglected and therefore our results only apply to the density profile of old stars. Furthermore, we use a simplified merger history

\footnotetext{
4 During our referee process, Jingade et al. (2015) presented new adiabatic growth models using classical Sérsic bulges for the initial setup of galaxies. Their models cover the other extreme, i.e., the least luminous galaxies with nuclear star clusters.
}

and assume that core profiles are created by the currently most accepted model, the SMBH binary evolution scenario. In the scenario of cosmic structure formation, several major merger events (4-5) are expected to occur between $z=0$ and $z=3$ (Conselice 2007) with a strong decline at $z=1$. Our models start at this redshift and we assume that core profiles were fully grown up to $z=1$, so we can use current parameter relations to fix the break radius. We neglect the $\approx 1$ major merger events since $z=1$. Alternatively, we assume that alternating phases of core creation through merging and adiabatic cusp regrowth are dynamically equivalent.

\subsection{N-body setup}

\subsubsection{Runs}

Nine representative SMBH growth models from Sect. 2 are selected for our $N$-body computations. On a logarithmic scale, they equally cover the whole parameter space including the extreme scenarios. The models are listed with their initial parameters in Table 1. The most extreme growth mode (Model 9) is only computed by means of $N$-body computations from redshift $z=1$ up to $z=0.1$. In this way, we enlarge the number of promising clusters hosting these massive SMBHs within our light cone.

Fully spin-powered models with $\epsilon=1$ (i.e., $\Delta M_{\bullet}=0$ ) are automatically computed in the form of our reference models. They do not lead to adiabatic cusp regrowth since all binding energy is radiated away. The conversion of given cosmic epochs into redshifts is calculated with the public available "Cosmological Calculator for the Flat Universe" from Nick Gnedin using $\Omega_{0}=0.3036$ and $H_{0}=68.14 \mathrm{~km} \mathrm{~s}^{-1} \mathrm{Mpc}^{-1}$.

\subsubsection{Initial galaxy profiles and parameters}

We use core-Sérsic models (Graham et al. 2003; Trujillo et al. 2004) for our initial galaxy setup since we assume that galactic cores are created within the first 5-6 Gyr, prior to the onset of AGN cluster feedback. The models are generated in equilibrium (including central black holes) with the procedure described in Brockamp et al. (2014). To exclude any dynamical instabilities related to anisotropic models (i.e., tangetially biased within $R_{\text {break }}$ and radially biased at large radii), we only 
use models with an isotropic velocity distribution. Following Graham et al. (2003) and Trujillo et al. (2004), the six parametric core-Sérsic models have a light profile, $I(r)$, (i.e., 2D density profile assuming a constant mass to light ratio) of the form,

$$
\begin{aligned}
I(R)= & I_{\mathrm{b}} 2^{-\frac{\gamma}{\alpha}} \exp \left[b\left(2^{\frac{1}{\alpha}} R_{\mathrm{b}} R_{\mathrm{e}}^{-1}\right)^{\frac{1}{n}}\right] \\
& \times\left[1+\left(\frac{R_{\mathrm{b}}}{R}\right)^{\alpha}\right]^{\frac{\gamma}{\alpha}} \exp \left\{-b\left[\frac{R^{\alpha}+R_{\mathrm{b}}^{\alpha}}{R_{\mathrm{e}}^{\alpha}}\right]^{\frac{1}{n \alpha}}\right\} .
\end{aligned}
$$

Here, $b$ is a normalization parameter such that half of the total luminosity is produced within the projected half-light radius, $R_{\mathrm{e}}$. The parameter, $b$, is obtained by numerically solving the integral Eq. (A10) in Trujillo et al. (2004). The Sérsic index, $n$, specifies the light concentration of the outer profile beyond the break radius, $R_{\text {break }}$, (for short $R_{\mathrm{b}}$ ) where the profile transforms into a power law with slope $\gamma$. The parameter $\alpha$ controls the sharpness of the transition and $I_{\mathrm{b}}$ is the light intensity at $R_{\mathrm{b}}$. Selected parameters for our models are listed in Table 1 and are discussed below.

By first fixing the initial SMBH mass at $M_{\bullet}\left[M_{9}\right]=$ $1.5,5,10,15,20,40, R_{\mathrm{b}}$ is then extracted from the parameter relation in Rusli et al. (2013). Although values as high as $R_{\mathrm{b}}=3.8 \mathrm{kpc}$ seem far-fetched, there exist central cluster galaxies, e.g., Abell 2261 and Abell 85, which have similar or even larger break-radii (Postman et al. 2012; López-Cruz et al. 2014). The effective radii, $R_{\mathrm{e}}$, and dynamical masses, $M_{\mathrm{dyn}}$, of our model galaxies are based on real galaxies (NGC 1399, M 60, NGC 3842, NGC 4889, NGC 6166) with similar black holes (Magorrian et al. 1998; Lauer et al. 2007b; McConnell \& Ma 2013). The SMBH masses $\left(M_{\bullet}\left[M_{9}\right]=15,20\right)$ are within the one sigma confidence limits of NGC 4889. Hence, NGC 4889 is used as a representative for our highest mass models. For the uppermost value $\left(M_{\bullet}\left[M_{9}\right]=40\right)$, we use NGC 6166. So far, NGC 6166 is the only source with a measured black hole mass based on stellar kinematics that is comparable to that limit (Magorrian et al. 1998). However, other studies have not yet confirmed the mass of NGC 6166. For galaxy models 8 and 9 , the bulge mass of NGC 6166 is rounded upward to $M_{\text {dyn }}\left[M_{9}\right]=2000$.

We use the central slope parameter $\gamma=0.2$, which is assumed to be shaped by SMBH merging. This value is in accordance with several observed core-type galaxies in Rusli et al. (2013) and Dullo \& Graham (2014) and it results in a 3D density profile, which declines faster than $r^{-0.5}$. This is an important constraint for isotropic models as it guarantees the dynamical stability within the influence radius of the black hole (Tremaine et al. 1994). The transition parameter, $\alpha=4.25$, is obtained from the arithmetic mean values from all galaxies in Rusli et al. (2013) and Dullo \& Graham (2014) except NGC 7768. This particular galaxy has a transition value that is too sharp, $\alpha \rightarrow \infty$, which would otherwise bias the arithmetic mean. All models and their parameters are summarized in Table 1.

\subsection{3. $N$-body computations}

The main computations are performed with the MUESLI software for collisionless dynamics (Brockamp et al. 2014) on six nodes of the VLBI-computer cluster at the Max Planck Institute for Radio Astronomy. The calculations lasted more than a month. MUESLI is a multi-purpose platform for the simulation of elliptical galaxies, their nuclear black holes, and (not relevant for this paper) globular clusters systems. As a check we also performed a few computations by direct $N$-body simulations using a self-customized version of NBODY6 with $N=5 \times 10^{5}$ particles (Aarseth 1999, 2003; Nitadori \& Aarseth 2012). This dual strategy allows us to exclude potential error sources. These are either related to two-body relaxation processes (relevant for direct summation codes) where particle numbers that are too small would lead to a cusp regeneration process around a central black hole (Bahcall \& Wolf 1976) or potential fluctuations induced by discreteness noise in the self-consistent field (SCF) method (Hernquist \& Ostriker 1992). The SCF formalism is implemented into the MUESLI software. We carefully tested (by finding a converging solution) the required particle number, $N$, and the required order of the radial and angular base functions. We use $10^{7}$ particles for the MUESLI simulations. Furthermore, to decrease the computational cost and to reduce numerical errors (i) Models 1 and 2 are not evolved for 850 (520), i.e., $8 \mathrm{Gyr}$ but for $50 \mathrm{~N}$-body time units only ( $\Delta M_{\bullet}$. remains unaffected). This strategy is applicable as we are only interested in adiabatic processes and want to suppress relaxation. (ii) During computations with MUESLI, the overall potential/density is not updated in Models 1-6 to reduce relaxation effects caused by discreteness noise. Model 7 is computed with (red dashed line) and without (red solid line) potential updates (PU). It is the model where back-reaction effects of the changing density profile (due to SMBH mass growth) start to become important. Therefore, Models 8 and 9 where $\Delta M_{\bullet}$ is of the order of the initial black hole mass itself are only computed with frequent updates of the potential.

Despite dynamical processes like SMBH wandering, dynamical heating, and relaxation-driven cusp formation, which occurs in NBODY6 computations (see, e.g., Brockamp et al. 2011), the agreement with the MUESLI simulations is nevertheless very good. The outcome of a NBODY6 computation is included in Fig. 3 (Model 9, solid blue line) for comparison. To exclude (as much as possible) even unknown systematics, we also compute all models without adiabatic black hole growth (i.e., with $\left.\Delta M_{\bullet}=0\right)$ and use them as a reference and for comparison. They also trivially correspond to fully spin-powered models. Finally, the amount of swallowed particles by the adiabatically growing black hole ranged from insignificant to small values when compared with the gas accretion rates and is therefore not discussed.

\subsection{Results}

Figure 3 shows the dynamical response of the projected (2D) density profiles to adiabatically growing SMBHs obtained with the MUESLI software (Brockamp et al. 2014). We use dimensionless model units, $R, \rho_{2 \mathrm{D}}$, for a better comparison. These models can be scaled to physical units by $R_{\text {phys }}=1.34 R_{\mathrm{e}} R$ and $\rho_{2 \mathrm{D}}\left(R_{\text {phys }}\right)=\rho_{2 D}(R) M_{\text {dyn }} /\left(1.34 R_{\mathrm{e}}\right)^{2} \propto \mu\left(R_{\text {phys }}\right)$, where $\mu$ is the surface brightness profile using an appropriate massto-light ratio. All computations (red curves for adiabatic models and black curves for reference models without growing $\mathrm{SMBH}$ ) contain $10^{7}$ particles. The resolution scale in Fig. 3 is $R_{\text {phys }}=16$ pc (Model 1) to $R_{\text {phys }}=270$ pc (Model 9). The central density slope, $\gamma$, is measured within half the initial break radius. Obtained uncertainties are based on assumed Poisson errors. Significant cusp regrowth is defined over the quantity $\Delta \gamma=\gamma_{\mathrm{ref}}-\gamma_{\mathrm{adiab}}$, which must be larger than the sum of the individual slope errors. Evidently, noticeable cusp growth is only observed in Models 5-9, which means that the black hole accreted more than $25 \%$ of its initial mass.

In Fig. 4 we plot the velocity distribution, $\beta=1-\left(\sigma_{\theta}^{2}+\right.$ $\left.\sigma_{\phi}^{2}\right) / 2 \sigma_{r}^{2}$, of Models 8 and 9. In very good agreement with 
M. Brockamp et al.: Unveiling Gargantua

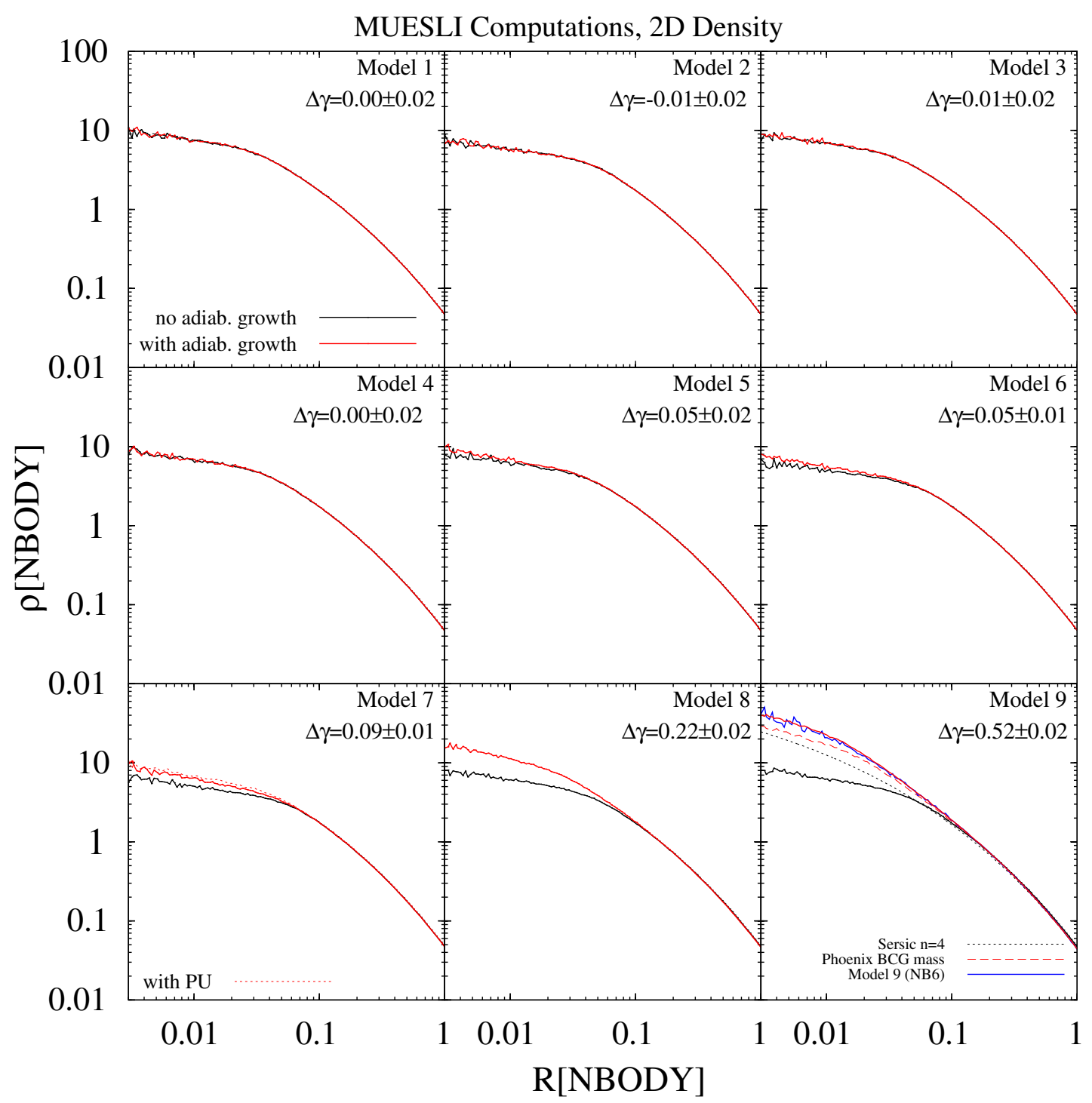

Fig. 3. Projected (2D) density profiles of all nine models. Red lines represent adiabatic SMBH growth models after $T=8$ Gyr (Models 1-8) and $T=6.7 \mathrm{Gyr}$ (Model 9), whereas black lines show reference models without growing SMBHs (i.e., $\Delta M \bullet=0$ ). The density slopes, $\gamma$, are fitted within half the size of the initial break radii by using a simple power-law approximation. Hence, they are influenced by the outer profile and we quote them with their sign. In ascending order of the computed reference Models $1-9$ these are: $\gamma_{\text {ref }}=$ $-0.26 ;-0.26 ;-0.3 ;-0.3 ;-0.28 ;-0.23 ;-0.25 ;-0.3 ;-0.3$. The quantity $\Delta \gamma=\gamma_{\text {ref }}-\gamma_{\text {adiab }} \geq 0$ results from a growing density cusp due to adiabatic SMBH growth. Evidently, accretion rates with $\Delta M_{\bullet} \gtrsim M_{\bullet}\left(t_{0}\right)$ lead to a cusp regeneration process. Smaller accretion rates (Models $\left.1-5\right)$ have no strong impact on the innermost 2D density slope. In panel 9 a pure Sersic $n=4$ model is added for comparison (black dotted line). The solid blue line corresponds to the NBODY6 computation of the same model. It is in excellent agreement with the solid red line. The red dashed line is similar, but is scaled to a 1.5 times larger host galaxy mass, similar to the Phoenix BCG (McDonald et al. 2013).

the outcomes of semianalytical approaches (e.g., Goodman \& Binney 1984, Quinlan et al. 1995, their Fig. 2) the isotropic velocity distribution around the pre-existing seed black hole, $\beta(R)=0$, changes. Particles on eccentric orbits are dragged more efficiently toward the innermost center where the velocity distribution stays isotropic. Slightly outside, a tangential biased region, which is dominated by particles on more circular orbits forms. This has consequences for the stellar dynamical black hole mass determination method. Extreme cool-core clusters like the Phoenix- or RX J1347.5-1145 cluster with rapidly growing SMBH masses (unless $\epsilon>0.1$ ) are rare within our light cone. At huge cosmic distances, the innermost isotropic region (if it has not already been destroyed by SMBH merging) might be out of the resolution scale and the region where circular orbits dominate is left. The measurable radial velocities are biased toward lower values and thus give rise to an underestimation of the true black hole mass if not properly taken into account. The effect is enhanced if the initial model already starts with a tangentially biased core as expected from SMBH scouring models.

\section{Discussion}

General results, the method to unveil the most massive central cluster SMBHs and the outlook are part of this section.

\subsection{How to unveil the most massive black holes and implications for scaling relations}

In Sect. 2 we compiled a set of SMBH growth rates, which are based on the mechanical feedback energies of central 


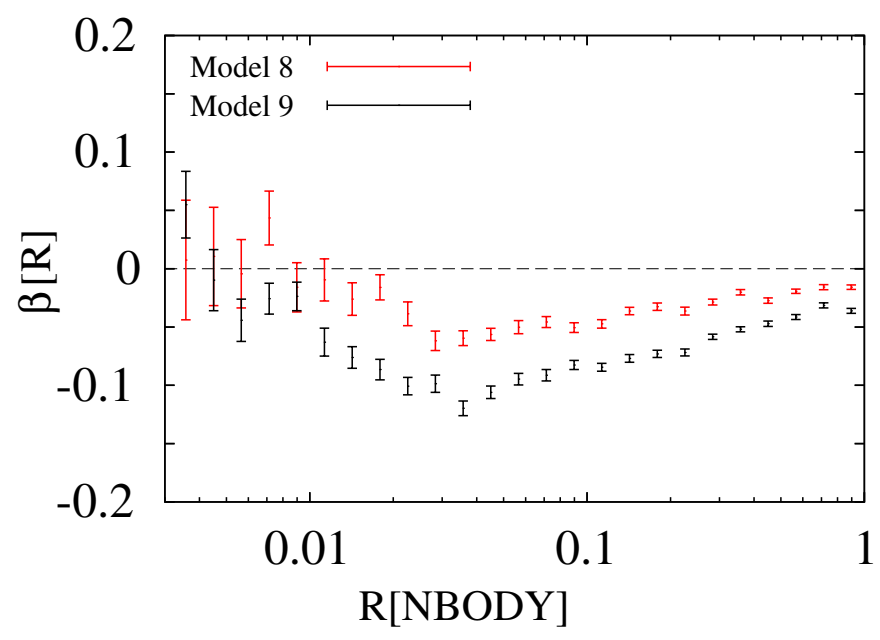

Fig. 4. (Analytical) initial (i.e., $\Delta M_{\bullet}\left[M_{9}\right]=0$, black dashed line) and final velocity distribution, $\beta=1-\left(\sigma_{\theta}^{2}+\sigma_{\phi}^{2}\right) / 2 \sigma_{r}^{2}$, of our most extreme Models 8 and 9 . We use the $3 \mathrm{D}$ radius for the evaluation of the velocity dispersion components, $\sigma_{\theta}, \sigma_{\phi}, \sigma_{r}$. Error values are obtained from the bootstrapping method. Particles on eccentric orbits are attracted more strongly toward the innermost center such that circular orbits, i.e., a tangential biased region develops slightly outside but still very close to the center. This effect is relevant for the stellar dynamical mass measurement of the central black hole.

cluster AGN. The adiabatic growth of hot cluster gas results in a cusp regrowth process if the ratio of mass gain to initial SMBH mass (at $z=1$ ) exceeds $25 \%$. This ratio is lowered if the initial slope is smaller than $\gamma=0.2$ or, more generally, if the total mass within the core is smaller than assumed for our computations. Nevertheless, SMBH growth in ordinary elliptical galaxies/BCGs (represented by our Models 1-4) should not lead to a significant adiabatic contraction. This may change in the most massive and X-ray luminous clusters with the highest feedback energies. Their central cluster black holes likely grow by several billion solar masses since redshift one unless most of the binding energy is radiated away $(\epsilon \approx 1)$. Assuming accretion efficiencies even below $\epsilon \approx 0.1$, sources with the highest feedback energies might even double their masses during the hot cluster gas accretion phase. Therefore, some nuclear black holes in the hottest and most X-ray luminous clusters might have accumulated (through hot gas accretion and SMBH coalescences) masses of the order of $10^{11} M_{\odot}$ (see Sect. 4.2 for some candidates). If the observation-based models (Eq. (4)) with small $\epsilon$ are realized in some of these galaxy clusters, even the most prominent cores will be healed or even replaced by steep density cusps. In Model 8 the initial core-Sérsic model is transformed back into a shape that resembles a classical Sérsic profile, whereas Model 9 develops a density cusp. This has consequences for the prediction power of the locally calibrated $M_{\bullet}-R_{\text {break }}, M_{\bullet}-R_{\text {cusp }}$ and $M_{\bullet}-L_{\text {def }}$ scaling relations. Black holes at the upper mass limit do not necessarily inhabit galaxies with the most pronounced light deficits/cores. Adiabatic SMBH growth could also affects the $M_{V}-R_{\text {cusp }}$ relation (Lauer et al. 2007a), which links the absolute $V$-band magnitude of a galaxy to the size of the cusp radius obtained by a Nuker-profile fit. As light deficits are decreased by adiabatic contraction, the most massive black holes might be located in BCGs with core-density profiles, which are less pronounced than expected from the total luminosity of their host galaxy. The first observational support for our prediction might already have been found in Savorgnan \& Graham (2015).
However, it is necessary to include additional, even more extreme cases to see whether their observed trend continues.

Finally, one can also argue that BCGs with huge break radii and light deficits like MS0735, which are known to be in a rapid mass accretion process (unless $\epsilon \approx 1$ ) (McNamara et al. 2009), must indeed be ultramassive, otherwise the ratio of $\Delta M_{\bullet}$ to the initial black hole mass would be too large and the core would have been replaced by a much steeper cusp. In this sense our computations also strengthen the case that galaxies with huge central cores witness the presence of ultramassive black holes. The ratio of black hole growth by merging (carving out shallow cores) to hot gas accretion (steepening core profiles) is crucial.

\subsection{Massive black hole candidates and a test}

Three promising galaxy clusters, which might host very massive black holes, are RX J1347.5-1145, SPT-CLJ2344-4243, (the Phoenix cluster) and Abell 2029. We now discuss how they are related to our results and why they should be used for constraining hot gas accretion models. Additionally, they might help to uncover the limitations of various galaxy-SMBH scaling relation.

- $R X$ J1347.5-1145: this object at redshift $z=0.45$ is one of the most X-ray luminous $\left(L_{X}=6 \times 10^{45} \mathrm{erg} \mathrm{s}^{-1}\right.$ in the [2-10] keV band) and massive clusters known (Gitti \& Schindler 2004). Depending on the accretion efficiency and assuming that its mechanical AGN feedback balances central gas cooling, as supported by Churazov et al. (2002), Gitti et al. (2007), its nuclear black hole might have swallowed several $10^{9} M_{\odot}$ of hot gas (or even more if $\epsilon<0.1$, Sect. 2.2). However, this is only one aspect. In their lensing analysis, Köhlinger \& Schmidt (2014) used 3D pseudoJaffe models to parameterize the two central galaxies. For the brightest cluster galaxy (BCG), they deduced a velocity dispersion around $\sigma \approx 600 \mathrm{~km} \mathrm{~s}^{-1}$ (a value that is also supported by gas spectroscopy, but might not necessarily result from a virialized state Sahu et al. 1998) and a 3D core radius in excess of a few kpc (it will be reduced in a $2 \mathrm{D}$ projection). If future observations confirm these values, the case for an extremely massive black hole in the core of RX J1347.51145 would be strengthened. It would require a black hole merger rate at the upper end of the predictions of cosmological rich-cluster simulations (Laporte \& White 2015) to carve out a kpc sized core in a galaxy with a huge velocity dispersion $^{5}$ and to protect it against core healing by hot gas accretion.

- Phoenix cluster: this cluster is even more massive and X-ray luminous than RX J1347.5-1145 (McDonald et al. 2012). It could be one of the few objects in the visible Universe where a model as extreme as our Model 9 might be realized and whose nuclear black hole might exceed $10^{11} M_{\odot}$. Based on this scenario and our initial $N$-body setup, we predict that the old/red stellar density profile of the Phoenix BCG (red curves in Fig. 3) should resemble a classic Sérsic $n=4$ model, which becomes slightly steeper and more cusp-like within $0.13 R_{\mathrm{e}}$. Interestingly, the inner most data points (within $\approx 0.1 R_{\mathrm{e}}$ ) of the measured surface brightness profile in Fig. 3 from McDonald et al. (2013) seem to lie above the Sérsic $n=4$ reference model. However, our

5 The black hole radius of influence, $R_{\text {infl }}$, decreases with growing velocity dispersion since $R_{\text {infl }} \propto \sigma^{-2}$. Also, the simulated clusters in Laporte \& White (2015) are several times less massive (even) at $z=0$ than RX J1347.5-1145 and SPT-CLJ2344-4243 at $z \approx 0.5$. 
$N$-body models are not fine-tuned to match the properties of the Phoenix BCG nor its redshift exactly. A more compact size of its BCG of $R_{\mathrm{e}}=17 \mathrm{kpc}$ (McDonald et al. 2013) but otherwise identical initial parameters results in a larger initial mass/light deficit. This would necessitate even larger amounts of swallowed mass, $\Delta M_{\bullet}$, to steepen its central slope. Likewise, the applied bulge mass of its BGC has an influence too, as shown by the two red curves in Fig. 3.

- Abell 2029: the central host galaxy, IC 1101, is listed as one of the optically most luminous and largest cD-galaxies (Dressler 1979; Lin \& Mohr 2004). Therefore, a merger driven growth of its central black hole at the upper limits $\left(M_{\bullet} \approx[5-7] \times 10^{10} M_{\odot}\right)$ of cosmological predictions (Laporte \& White 2015) seems reasonable. Additionally, IC 1101 is embedded within a massive and relaxed galaxy cluster (Lewis et al. 2003). If classical Bondi accretion with its quadratical SMBH mass dependency (Bondi 1952, our Sect. 2.3) is realized, the nuclear SMBH should accrete at high levels. A precisely measured core size/light deficit might help to measure the ratio of SMBH scouring to adiabatic growth in one of the most extreme galaxies.

Finally, López-Cruz et al. (2014) report on the BCG in the Abell 85 cluster. So far it is the most extreme representative of a core-type galaxy with a measured break radius (cusp radius) of $R_{\mathrm{b}}=20 \mathrm{kpc}\left(R_{\mathrm{c}}=4.6 \mathrm{kpc}\right)$ (López-Cruz et al. $2014)^{6}$. Based on the extrapolation of the $M_{\bullet}-R_{\text {break }}, M_{\bullet}-R_{\text {cusp }}$ and $M_{\bullet}-L_{\text {def }}$ relations, its central black hole might also have a mass around $10^{11} M_{\odot}$ (López-Cruz et al. 2014).

It would be highly informative and important for constraining the limitations of gas accretion- and SMBH merging processes, especially with respect to their cluster environments, if the nuclear black hole masses in these four objects could be measured and compared with empirical scaling relations. With this approach, our prediction that the truly most massive black holes are not necessarily located in galaxies with the largest cores could be put to its first test.

\subsection{Outlook}

We now discuss future applications with the black hole mass gain sensitive calorimeter, how its sensitivity can be increased, and describe a nonfeedback regulated SMBH growth channel, which under the right circumstances can be realized in the hottest and most massive galaxy clusters.

\subsubsection{Future applications}

A robust calorimeter requires detailed information about initial conditions. With statistically meaningful knowledge about central surface brightness profiles of BCGs at the onset of hot gas accretion, in combination with deposited AGN feedback energies, the calorimeter can be used to determine the significance of cold (during the quasar phase) and hot gas accretion as well as SMBH merging. It also offers a tool to measure the accretion efficiency parameter. The calorimeter method is based on photometric, instead of spectral, imaging, therefore it is much easier to apply than the direct measurement of SMBH masses at these redshifts. A precisely calibrated SMBH mass gain sensitive calorimeter offers the potential to constrain the growth history of individual galaxies and their central black holes.

\footnotetext{
6 Bonfini et al. (2015) take a different position, showing that the stellar profile of the BCG inside Abell 85 can be well fitted with a classical Sérsic model without the need for a depleted core.
}

We want to illustrate this idea on the basis of NGC 4889 and NGC 4874, the central dominating galaxies in the Coma cluster. While NGC 4889 is indeed the brightest member, it has a nearly flat density core with a slope $\gamma=0.03$ and a break radius of $R_{\mathrm{b}}=970 \mathrm{pc}$ (Lauer et al. 2007b). Because of the flatness of its innermost surface profile, we do not expect, on the basis of the results presented in this paper, a significant phase of hot gas accretion throughout its life. Its central black hole of $M_{\bullet}=(6-37) \times 10^{9} M_{\odot}($ McConnell et al. 2011) was likely grown during a massive quasar phase, whereas subsequent SMBH merging activity has later shaped its core. On the other hand, NGC 4874 has a much more diffuse appearance and is thus classified as a cD-type galaxy. If we apply our results to this galaxy, we find a different history. Its central black hole was not formed during a massive quasar phase, but instead by the merger of several very massive progenitor galaxies. This gave rise to its extended halo as well as a very large break radius of $R_{\mathrm{b}}=1730$ pc (Lauer et al. 2007b) formed by SMBH scouring. Its central cusp profile, $\gamma=0.12$, is steeper than that of NGC 4889. Assuming its initial profile was flatter in the past (e.g., comparable to that of NGC 4889), hot gas accretion was likely relevant in this galaxy. The recent infall of the galaxy group dominated by NGC 4889 (Gerhard et al. 2007) maybe quenched its activity through the disruption of the accretion flow. We can estimate the central SMBH mass in NGC 4874 from its break radius, the empirical $M_{\bullet}-R_{\mathrm{b}}$ relation given in Rusli et al. (2013), and add the mass required to increase its central slope by $\Delta \gamma=0-0.09$ (taken from our Model 7). However, the value $\Delta M_{\bullet}\left[M_{9}\right] \approx 10$ relevant for the highest increase $\Delta \gamma=0.09$ must been taken as an upper limit: the measured slope in NGC 4874 is even flatter than $\gamma=0.2$ (which is chosen for our galaxy computations) and the dynamical mass within its core is smaller (due to its more extended size and flatter slope) than assumed for our reference Model 7. Consequently, even moderate hot gas accretion with a value around $\Delta M_{\bullet}\left[M_{9}\right] \approx 1$ might have shaped the central slope of NGC 4874. Its nuclear black hole might therefore be as massive as $M_{\bullet} \approx(20-30) \times 10^{9} M_{\odot}$ with a tendency toward the lower value, making it comparable to its neighbor. This mass estimate is also compatible with dynamical mass measurements performed in Magorrian et al. (1998).

\subsubsection{Potential oscillations}

In our computations we assume that initial core formation proceeds via well-established SMBH merger scenarios and that the cores are fully grown at the onset of hot cluster gas accretion. In contrast to this assumption Martizzi et al. (2012, 2013) show, that based on idealized computations (e.g., final SMBH masses following $M_{\bullet}-\sigma$ ), centrally flattened profiles can also be produced by oscillations of the central potential induced by repetitive AGN outbursts. However, their models predict core sizes much in excess of those typically observed (Dullo \& Graham 2014). Nevertheless, we hope that future studies that aim to investigate the influence of hot cluster gas accretion onto the most massive black holes in giant cool core galaxy clusters consider both scenarios: (i) Adiabatic cusp formation by hot gas accretion, which is not restricted by the $M_{\bullet}-\sigma$ relation (see, e.g., Hlavacek-Larrondo et al. 2012a, and the results obtained in this paper); and (ii) the destruction of density cusps by potential oscillations. Without the cold clumps, used in Martizzi et al. (2013) to mimic infalling galaxies and whose extended gas halos might be stripped more efficiently in hotter and more massive galaxies clusters, core sizes might be reduced due to less violent potential fluctuations (Martizzi et al. 2013). With smaller initial cores 
created by potential oscillations, adiabatic cusp formation by hot gas accretion might dominate at least in the most massive clusters hosting the most violently growing SMBHs. Core creation by potential fluctuations can also be implemented in future versions of the MUESLI software. In addition to the stellar/dark matter (DM) profile, a particle distribution that represents the density profile of the ambient gas can be generated in equilibrium. These gas particles are not dynamically evolved forward in time but their masses are temporarily lowered within (e.g., bipolar) volumes to mimic cavities whose size depends on the equation of state of the gas and jet power. Depending on additional parameters, such as the duty cycle of the AGN, longevity of cavities, and the fraction of jet energy, which goes into cavity production and shock generation, the stellar/DM particles (which are dynamically evolved forward in time) will feel these potential oscillation and react correspondingly. In addition, the mass of the black hole is adiabatically increased, as already studied in this paper. The implemented angular base functions (spherical harmonics) already have a topology, which strongly resembles observed cavity systems. Furthermore, the high-resolution scale of MUESLI allows us to study the impact of potential oscillations deeply within the influence radius of the black hole, making it a reliable alternative to grid-based codes.

\subsubsection{SMBH runaway growth}

In Sect. 2.3 we compared observed AGN feedback energies with those obtained from the prediction of classical Bondi models. For the sake of simplicity, we assumed that the growing black hole has no influence on the accretion rate over typical cluster lifetimes of $8 \mathrm{Gyr}$. However, because of increasing evidence of the existence of black holes in excess of $10^{10} M_{\odot}$ and the generic $\mathrm{d} M_{\bullet} / \mathrm{d} t=\gamma M_{\bullet}^{2}$ dependency of classical Bondi accretion, this assumption might turn out to be wrong, at least for a few extreme objects. From a formal perspective, Bondi accretion should amplify itself and accelerate ${ }^{7}$ as

$$
M_{\bullet}(t)=\frac{M_{\bullet}(t=0)}{1-\gamma M_{\bullet}(t=0) t} .
$$

However, in reality the accretion rate does not diverge. First of all, (i) the gas reservoir is finite; (ii) the Bondi accretion parameters, which are expressed as $\gamma$, are not necessarily constant over time subject to AGN feedback; and (iii) by deriving the classical Bondi accretion rate (Eq. (5)), one has to assume that the mass within the loss cone is small compared to the mass of the accretor. Nevertheless, via Eq. (7) and the initial parameters from Sect. 2.3 with a black hole mass around $4 \times 10^{10} M_{\odot}$, the accretion rate is still expected to accelerate. After several billion years it would exceed a few percent of the Eddington rate where it likely changes to a radiatively efficient accretion state (Gallo et al. 2003; Maccarone 2003; Falcke et al. 2004; Wu \& Cao 2008). Depending on the broadband spectrum of the quasar $^{8}$ and the gas temperature of the cluster, it might either lead to inverse Compton cooling (Fabian \& Crawford 1990), which decreases the gas temperature and would stimulate even higher accretion rates, or to Compton heating (Sazonov et al. 2004, 2005). Interestingly, powerful quasars with luminousities around $L \approx 10^{47}-10^{48} \mathrm{erg} \mathrm{s}^{-1}$ (comparable to our model predictions) are reported to be located in the Phoenix (Ueda et al. 2013) and CL1821+643 cluster (Walker et al. 2014). These are two of the strongest cool-core and most massive galaxy clusters.

\footnotetext{
7 We call it SMBH runaway growth as it is mathematically equivalent to a different scenario proposed in Hernandez \& Lee (2010).

8 Thanks to our referee for pointing this out.
}

\section{Summary}

This paper is about a new strategy to unveil the most massive black holes in the Universe. SMBHs at the highest mass scale are important for constraining the limitations of empirical scaling relations, which are useful for theoretical model building. With growing evidence that the most massive black holes are located at the centers of very massive galaxy clusters (e.g., Hlavacek-Larrondo et al. 2012a), we present a simple and effective method to pick out the most extreme candidates.

For that purpose, we first transformed (observed) AGN feedback powers into central cluster SMBH growth rates (Sect. 2). Through a comparison with classical Bondi models we checked whether the initial black hole masses adopted for our $N$-body computations are reasonable. We found a strong overlap between theoretically predicted and observationally observed feedback powers. This indicates that simple Bondi accretion (even when adapted for rotating gas atmospheres) can fuel the most powerful cluster AGN.

In the main part of our paper (Sect. 3), we computed with the help of the MUESLI software the dynamical response of the host galaxy to the growing SMBH. With this approach, we constructed a black hole mass gain sensitive calorimeter. In principle, a precisely calibrated calorimeter offers a way to measure the contribution of cold gas accretion, SMBH merging and subsequent hot cluster gas accretion to the growth of the central cluster black hole. For our initial $N$-body setup, we used core-Sérsic models to account for SMBH scouring as well as nine different SMBH growth scenarios, encompassing in logarithmically increasing steps the whole parameters space of compiled gas accretion models. We found that adiabatically driven cusp formation is significant in the most extreme growth models, but can be neglected if the ratio of accreted hot gas to initial black hole mass is around $25 \%$ or smaller. Hence, our results also confirm the expectation that galaxies with large central core profiles (especially if they are located in galaxy clusters with huge deposited feedback energies and assuming they are not mainly spin powered) witness the presence of extremely massive black holes. Otherwise, the core would have been replaced by a steeper central slope. However, in the most X-ray luminous galaxy clusters with permanent AGN feedback powers as high as $\left(P_{\mathrm{AGN}} \approx 10^{46} \mathrm{erg} \mathrm{s}^{-1}\right)$ even the most pronounced initial cores will be turned into cusps if accretion efficiencies are smaller than $\epsilon=0.1$. We argue that the nuclear black holes inside RX J1347.5-1145, the Phoenix cluster, and Abell 2029 might be among the most massive black holes in the Universe. Secure mass measurements of their central black holes would not only help to uncover limitations of scaling relation but could be used to constrain the limitations of gas accretion and SMBH merging processes. They also might represent promising candidates for future applications with the Event Horizon Telescope (Doeleman et al. 2009). The hunt for Gargantua is on.

Acknowledgements. We thank Walter Alef and Helge Rottmann for their technical support with the VLBI-computer cluster and Olaf Wucknitz and Vladimir Karas for helpful comments. Special thanks go to Stefanie Komossa for her readiness to improve the quality of this manuscript and for the instructive discussions about black hole physics. Finally, we would like to thank our referee.

\section{References}

Aarseth, S. J. 1999, PASP, 111, 1333

Aarseth, S. J. 2003, Gravitational N-Body Simulations (Cambridge University Press)

Allen, S. W., Dunn, R. J. H., Fabian, A. C., Taylor, G. B., \& Reynolds, C. S. 2006, MNRAS, 372, 21 
Banerji, M., Alaghband-Zadeh, S., Hewett, P. C., \& McMahon, R. G. 2015, MNRAS, 447, 3368

Bahcall, J. N., \& Wolf, R. A. 1976, ApJ, 209, 214

Bîrzan, L., Rafferty, D. A., Nulsen, P. E. J., et al. 2012, MNRAS, 427, 3468

Bondi, H. 1952, MNRAS, 112, 195

Bonfini, P., Dullo, B. T., \& Graham, A. W. 2015, ApJ, 807, 136

Brockamp, M., Baumgardt, H., \& Kroupa, P. 2011, MNRAS, 418, 1308

Brockamp, M., Küpper, A. H. W., Thies, I., Baumgardt, H., \& Kroupa, P. 2014, MNRAS, 441, 150

Cavagnolo, K. W., McNamara, B. R., Nulsen, P. E. J., et al. 2010, ApJ, 720, 1066

Chaudhuri, A., Nath, B. B., \& Majumdar, S. 2012, ApJ, 759, 87

Churazov, E., Sunyaev, R., Forman, W., Boehringer, H. 2002, MNRAS, 332, 729

Conselice, C. J. 2007, IAU Symp., 235, 381

Doeleman, S., Agol, E., Backer, D., et al. 2009, in Astro2010: The Astronomy and Astrophysics Decadal Survey, 68

Dressler, A. 1979, ApJ, 231, 659

Dullo, B. T., \& Graham, A. W. 2014, MNRAS, 444, 2700

Dunn, R. J. H., \& Fabian, A. C. 2006, MNRAS, 373, 959

Ebisuzaki, T., Makino, J., \& Okumura, S. K. 1991, Nature, 354, 212

Fabian, A. C. 2012, ARA\&A, 50, 455

Fabian, A. C., \& Crawford, C. S. 1990, MNRAS, 247, 439

Falcke, H., Körding, E., \& Markoff, S. 2004, A\&A, 414, 895

Ferrarese, L., \& Merritt, D. 2000, ApJ, 539, L9

Frank, J., King, A., \& Raine, D. 1992, Accretion power in astrophysics, Camb. Astrophys. Ser., 21

Gallo, E., Fender, R. P., \& Pooley, G. G. 2003, MNRAS, 344, 60

Gebhardt, K., Bender, R., Bower, G., et al. 2000, ApJ, 539, L13

Gebhardt, K., Adams, J., Richstone, D., et al. 2011, ApJ, 729, 119

Gerhard, O., Arnaboldi, M., Freeman, K. C., et al. 2007, A\&A, 468, 815

Ghisellini, G., Foschini, L., Volonteri, M., et al. 2009, MNRAS, 399, L24

Gitti, M., \& Schindler, S. 2004, A\&A, 427, L9

Gitti, M., Piffaretti, R., \& Schindler, S. 2007, A\&A, 472, 383

Goodman, J., \& Binney, J. 1984, MNRAS, 207, 511

Graham, A. W., Erwin, P., Trujillo, I., \& Asensio Ramos, A. 2003, AJ, 125, 2951

Gültekin, K., Richstone, D. O., Gebhardt, K., et al. 2009, ApJ, 698, 198

Häring, N., \& Rix, H.-W. 2004, ApJ, 604, L89

Hasinger, G., Miyaji, T., \& Schmidt, M. 2005, A\&A, 441, 417

Hawley, J. F., \& Krolik, J. H. 2006, ApJ, 641, 103

Hernandez, X., \& Lee, W. H. 2010, MNRAS, 404, L6

Hernquist, L., \& Ostriker, J. P. 1992, ApJ, 386, 375

Hlavacek-Larrondo, J., \& Fabian, A. C. 2011, MNRAS, 413, 313

Hlavacek-Larrondo, J., Fabian, A. C., Edge, A. C., \& Hogan, M. T. 2012a, MNRAS, 424, 224

Hlavacek-Larrondo, J., Fabian, A. C., Edge, A. C., et al. 2012b, MNRAS, 421, 1360

Hlavacek-Larrondo, J., McDonald, M., Benson, B. A., et al. 2015, ApJ, 805, 35

Jahnke, K., \& Macciò, A. V. 2011, ApJ, 734, 92

Jingade, N., Deep Saini, T., \& Tremaine, S. 2015, ApJ, submitted [arXiv: 1508.06409]

Jordán, A., Côté, P., West, M. J., et al. 2004, AJ, 127, 24

Jun, H. D., Im, M., Lee, H. M., et al. 2015, ApJ, 806, 109

Kelly, B. C., \& Shen, Y. 2013, ApJ, 764, 45

Körding, E. G., Jester, S., \& Fender, R. 2008, MNRAS, 383, 277

Köhlinger, F., \& Schmidt, R. W. 2014, MNRAS, 437, 1858

Kormendy, J., \& Bender, R. 2009, ApJ, 691, L142

Kormendy, J., \& Ho, L. C. 2013, ARA\&A, 51, 511

Kormendy, J., \& Richstone, D. 1995, ARA\&A, 33, 581

Laporte, C. F. P., \& White, S. D. M. 2015, MNRAS, 451, 1177

Lauer, T. R., Faber, S. M., Richstone, D., et al. 2007a, ApJ, 662, 808
Lauer, T. R., Gebhardt, K., Faber, S. M., et al. 2007b, ApJ, 664, 226 Lewis, A. D., Buote, D. A., \& Stocke, J. T. 2003, ApJ, 586, 135 Lin, Y.-T., \& Mohr, J. J. 2004, ApJ, 617, 879

Liu, F. K., \& Chen, X. 2013, ApJ, 767, 18

López-Cruz, O., Añorve, C., Birkinshaw, M., et al. 2014, ApJ, 795, L31

Ma, C.-J., McNamara, B. R., \& Nulsen, P. E. J. 2013, ApJ, 763, 63

Maccarone, T. J. 2003, A\&A, 409, 697

Magorrian, J., Tremaine, S., Richstone, D., et al. 1998, AJ, 115, 2285

Marconi, A., \& Hunt, L. K. 2003, ApJ, 589, L21

Martizzi, D., Teyssier, R., Moore, B., \& Wentz, T. 2012, MNRAS, 422, 3081

Martizzi, D., Teyssier, R., \& Moore, B. 2013, MNRAS, 432, 1947

Mathews, W. G., \& Guo, F. 2011, ApJ, 738, 155

Mathews, W. G., \& Guo, F. 2012, ApJ, 754, 154

McConnell, N. J., \& Ma, C.-P. 2013, ApJ, 764, 184

McConnell, N. J., Ma, C.-P., Gebhardt, K., et al. 2011, Nature, 480, 215

McDonald, M., Bayliss, M., Benson, B. A., et al. 2012, Nature, 488, 349

McDonald, M., Benson, B., Veilleux, S., Bautz, M. W., \& Reichardt, C. L. 2013, ApJ, 765, L37

McLure, R. J., \& Dunlop, J. S. 2002, MNRAS, 331, 795

McNamara, B. R., Kazemzadeh, F., Rafferty, D. A., et al. 2009, ApJ, 698, 594

McNamara, B. R., Rohanizadegan, M., \& Nulsen, P. E. J. 2011, ApJ, 727, 39

Merloni, A., \& Heinz, S. 2008, MNRAS, 388, 1011

Merritt, D. 2006, ApJ, 648, 976

Milosavljević, M., \& Merritt, D. 2001, ApJ, 563, 34

Narayan, R., \& Fabian, A. C. 2011, MNRAS, 415, 3721

Nitadori, K., \& Aarseth, S. J. 2012, MNRAS, 424, 545

Novikov, I. D., \& Frolov, V. P. 1989, Physics of black holes (Kluwer Academic Publishers)

Postman, M., Lauer, T. R., Donahue, M., et al. 2012, ApJ, 756, 159

Ravindranath, S., Ho, L. C., \& Filippenko, A. V. 2002, ApJ, 566, 801

Rusli, S. P., Erwin, P., Saglia, R. P., et al. 2013, AJ, 146, 160

Russell, H. R., McNamara, B. R., Edge, A. C., et al. 2013, MNRAS, 432, 530

Quinlan, G. D., Hernquist, L., \& Sigurdsson, S. 1995, ApJ, 440, 554

Sahu, K. C., Shaw, R. A., Kaiser, M. E., et al. 1998, ApJ, 492, L125

Savorgnan, G. A. D., \& Graham, A. W. 2015, MNRAS, 446, 2330

Sazonov, S. Y., Ostriker, J. P., \& Sunyaev, R. A. 2004, MNRAS, 347, 144

Sazonov, S. Y., Ostriker, J. P., Ciotti, L., \& Sunyaev, R. A. 2005, MNRAS, 358, 168

Silk, J., \& Rees, M. J. 1998, A\&A, 331, L1

Simionescu, A., Werner, N., Urban, O., et al. 2013, ApJ, 775, 4

Thomas, J., Saglia, R. P., Bender, R., Erwin, P., \& Fabricius, M. 2014, ApJ, 782, 39

Thorne, K. S. 1974, ApJ, 191, 507

Tichy, W., \& Marronetti, P. 2008, Phys. Rev. D, 78, 081501

Trakhtenbrot, B. 2014, ApJ, 789, L9

Tremaine, S., Richstone, D. O., Byun, Y.-I., et al. 1994, AJ, 107, 634

Trujillo, I., Erwin, P., Asensio Ramos, A., \& Graham, A. W. 2004, AJ, 127, 1917

Ueda, S., Hayashida, K., Anabuki, N., et al. 2013, ApJ, 778, 33

van den Bosch, R. C. E., Gebhardt, K., Gültekin, K., et al. 2012, Nature, 491, 729

Vantyghem, A. N., McNamara, B. R., Russell, H. R., et al. 2014, MNRAS, 442, 3192

Walker, S. A., Fabian, A. C., Russell, H. R., \& Sanders, J. S. 2014, MNRAS, 442, 2809

Wu, Q., \& Cao, X. 2008, ApJ, 687, 156

Wu, X.-B., Wang, F., Fan, X., et al. 2015, Nature, 518, 512

van der Marel, R. P. 1999, AJ, 117, 744

Vasiliev, E. 2014, Class. Quant. Grav., 31, 244002

Young, P. 1980, ApJ, 242, 1232 